\title{
Developing Cognitive and Emotional Trust Scale (CTS-6 \& ETS-6 in Supervisor) with Chinese and Japanese Sample
}

\author{
Pei LIU ${ }^{1, a}$, Wan-Fen GUO ${ }^{2, b, *}$ \\ ${ }^{1}$ North China University of Water Resources and Electric Power, Zhengzhou 450046, CHINA \\ ${ }^{2}$ The International College, Xiamen University, Xiamen 361102, Fujian, CHINA \\ 1'delicatemechanics@163.com, ²guowanfen@xmu.edu.cn. *Corresponding author
}

Keywords: Cognitive \& Emotional Trust, Social Exchange Theory, Employee-Supervisor Relationship, Scale development and validation.

\begin{abstract}
Our first purpose is to develop a general conceptualization of employees' cognitive and emotional trust in supervisor, which is systematic and detailed enough to predict how they causally relate to other constructs differently. Drawing on previous study, we proposed two central ideas based on which the two kinds of trust were conceptualized: (1) Cognitive trust functions to secure the productivity of social exchange relationship (SER) by focusing on the extrinsic utility of the exchange outcomes, from the perspective of gains and losses; (2) Emotional trust functions to build up a base of the long-term relationship by focusing on the intrinsic happiness inherent in the relationship and/or exchanging process, from the perspective of maintaining SER. Five middle-range hypotheses in organizational setting derived from this conceptualization were supported by Chinese and Japanese sample. Our second purpose is to develop and validate cognitive \& emotional trust scale in supervisor (CTS-6 \& ETS-6 in Supervisor), which can be distinguishable enough to reliably capture their unique relations in organizational setting across different cultures. Reliability, factorial validity, convergent \& discriminant validity, and criterion validity of CTS-6 \& ETS-6 in Supervisor were established by Chinese and Japanese sample.
\end{abstract}

\section{Introduction}

Organizational behavior studies have focused on two kinds of interpersonal trust-cognitive and emotional trust (or in other words, character- and relationship-based trust) - that may play key roles in ongoing work groups [1, 2]. Rousseau et al.'s cross-discipline review identified two key elements in various trust definitions: positive expectations and willingness to be vulnerable [3]. Under high-risk and high-interdependence situations, these elements make trust still effective in bringing out trustor's risk taking behaviors. Whereas cognitive trust is defined as such expectations and willingness based on the knowledge of trustee's character, emotional trust refers such expectations and willingness rooted in a high-quality long-term relationship with trustee [1, 2].

Although previous research is increasingly converging on the conceptualization of cognitive and emotional trust, two issues have been overlooked. First, existing conceptualization of them is not systematic and detailed enough to theoretically explain their unique relations with other constructs; second, existing measures of them are not distinguishable enough to reliably capture such unique relations across different cultures. This study aims to deal with these two issues.

\section{Rationale}

This work conceptualizes cognitive \& emotional trust based on the framework of social exchange theory $[4,5,6]$. We first provide some basic terms describing social exchange relationship (SER). According to [5], SER consists of exchanging process and long-term relationship.

Exchanging process is composed of three elements. The first is resources of exchange that can fall into two categories: objective resources with general utility and subjective resource with personal happiness, consistent with extrinsic-intrinsic incentives in motivation theory. The exchanges of 
objective resources are often conditioned by situations, whereas the exchanges of subjective resource are usually not. The second is exchanging behaviors referring to trustor's behaviors that exchange objective and subjective resource with trustee under reciprocity principle. The third is outcomes of exchange defined as the objective resources resulting from exchanging behaviors with a time lag and uncertainty. Thus, by definition whether exchanging behaviors bring a satisfactory outcome is usually situation-dependent. We do not use the term "outcome" to refer to subjective resource because it is a kind of intrinsic happiness inherent in exchanging behaviors.

Long-term relationship can be characterized by emotional bonds, the shared interests and values, and mutual obligations. In this work, we used the two kinds of ongoing trust to capture such relationship. Generally speaking, cognitive trust captures trust in trustee's characters, whereas emotional trust catches trust in the quality of this relationship [2]. Thus, we propose:

H1: In ongoing relationships interpersonal trust can be generally categorized into two different kinds: cognitive \& emotional trust (or in other words, character- \& relationship-based trust)

\section{Cognitive Trust in Supervisor}

Drawing on the arguments in literature of social exchange [4, 6], we propose the first central idea: (1) Cognitive trust functions to secure the productivity of SER by focusing on the extrinsic utility of the exchange outcomes, from the perspective of gains and losses. Some general properties of its underlying mechanism can be derived from this idea.

First, one basic part of cognitive trust mechanism is objective resource-based interdependence, such as employees' task \& management dependence on their supervisor. For example, cognitive trust mechanism would be deeply involved in the exchanges of objective resources, such as the opportunities to achieve high performance \& growth in work setting. For employees, the probability that they can obtain such opportunities usually depends on their supervisor's intention and, the potential of performance \& growth that they can achieve usually depends on the supervisor's ability. Thus, there is asymmetry interdependence in task \& management between employees and their supervisor. In general, objective resource-based interdependence yields the necessity for trustor to form the outcome-oriented expectations.

Second, such outcome-oriented expectations tend to focus on trustee decisions/actions that will provide trustor with extrinsic interests. For instance, employees can form the concrete expectations for their supervisor's decisions/actions in specific situations, by combining the cognition of the supervisor's characters and the understanding of task \& management dependence structure. Three most important characters - (a) competence, (b) benevolence, and (c) integrity - in work setting can be specified from prior study [7], which reflect three basic content domains of these expectations. Because such outcome-oriented expectations are involved in judging whether the objective resource exchanges with supervisor will be beneficial or harmful, cognitive trust can serve to secure the productivity of SER.

Finally, from the perspective of gains and losses, the main antecedent of cognitive trust is the past outcomes of objective resource exchanges, such as the opportunities that employees obtained from the supervisor in the past, rather than the exchanging behaviors for subjective resource (e.g., relationship-oriented altruistic behavior for supervisor). To secure the productivity of SER, cognitive trust mechanism requires an estimating process, by which employees can combine the past outcomes with the understanding of task \& management dependence structure to estimate the supervisor's characters [2]. For example, employees can use the potential of the obtained opportunities to estimate the supervisor's competence, and use the number of the obtained opportunities to estimate his/her benevolence. Thus, we propose following middle-range hypotheses in organizational setting:

H2-a: Opportunities obtained in the past has positive effect on cognitive trust in supervisor (convergent validity hypothesis);

H2-b: This effect is stronger than the effect of employees' altruistic behavior for supervisor on cognitive trust in supervisor (discriminant validity hypothesis). 


\section{Emotional Trust in Supervisor}

Based on the arguments in social exchange literature [5, 6], we propose the second central idea: (2) Emotional trust functions to build up a base of the long-term relationship by focusing on the intrinsic happiness inherent in the relationship and/or exchanging process, from the perspective of maintaining SER. Following general properties of its underlying mechanism can be inferred according to this idea.

First, one basic part of emotional trust mechanism is subjective resource-based interdependence, such as work life happiness interdependence between employees and their supervisor. Emotional trust mechanism would be involved closely in the exchanges of subjective resource, such as the warmth \& happiness inherent in relationship-oriented altruistic behaviors. For example, employees and the supervisor have long worked together in a work group, so that the interactions between them are inevitably frequent, which in turn would lead to such symmetrical interdependence of happiness in their work life. Generally, such subjective resource-based interdependence causes necessity for trustor to generate the relationship-oriented expectations/willingness.

Second, the relationship-oriented expectations/willingness tend to focus on trustor-trustee interactions in which the intrinsic happiness inherent. Three key aspects of the long-term relationship - (d) emotional bonds, (e) the shared long-term interests and values, and (f) mutual obligations - can be identified from previous literature, which mirror three basic content domains of these expectations/willingness. Since such relationship-oriented contents are relevant to a sense of how comfortable the relationship is, emotional trust can bring out subjective resource exchanges (e.g., reciprocal care and concern), which in turn would strengthen the relationship.

Finally, from the perspective of maintaining SER, one major antecedent of emotional trust is exchanging behaviors for subjective resource, such as employees' relationship-oriented altruistic behaviors, rather than the past outcomes of objective resource exchanges (e.g., opportunities obtained in the past). To long maintain SER, emotional trust mechanism requires a relationship-building process, by which exchanging behaviors for subjective resource can build up a base of the relationship, independently of what short term outcomes the objective resource exchanges lead to. For instance, employees' relationship-oriented altruistic behaviors can further the relationship by (d) increasing the amount of empathy to strengthen the emotional bonds; (e) increasing the amount of perspective taking to further the development of the common interests and values; and (f) deepening the mutual obligation and gratitude in the relationship. Thus, we propose two middle-range hypotheses in organizational setting:

H3-a: Employees' altruistic behavior for supervisor has positive effect on emotional trust in supervisor (convergent validity hypotheses);

$H 3-b$ : This effect is stronger than the effect of opportunities obtained in the past on emotional trust in supervisor (discriminant validity hypothesis).

\section{Method}

\section{Item Generation}

To generate an initial pool of items, at step1, we identified six content domains being consistent with the guidelines described above: (a) competence, (b) benevolence, (c) integrity, (d) emotional linkage, (e) identification, and (f) depending willingness. At step 2, we composed the initial version of item pool with items collecting from 17 existing trust measures, and then classified these items following content domains of each construct. At step 3, we generated some original items based on content domains of each construct. As a result, the item pool turned into a version of 94 items, which were then translated into Chinese and Japanese version separately.

\section{Content Validity}

Five Chinese managers and three Chinese management researchers evaluated Chinese version of these 94 items. Items were eliminated if, upon further consideration, (1) they described situations not 
common to most organizations, or (2) their contents did not correspond to the definitions, or (3) they would imply sensitive issues for Chinese organizational contexts. In this process, no item was eliminated. And then, five Japanese organizational behavior experts (three researchers and two Ph.D. students) assessed Japanese version of these 94 items using the same procedure. As a result, 6 items of (f) depending willingness domain were eliminated because they would imply sensitive issues for Japanese organizational contexts. Therefore, the final version of item pool consists of 88 items, with the following content domain distribution: (a) competence, 19 items; (b) benevolence, 9 items; (c) integrity, 15 items; (d) emotional linkage, 14 items; (e) identification, 16 items; (f) depending willingness, 15 items.

\section{Samples and Procedures}

Sample 1 (88 trust items). To empirically explore the factor structure of the 88 items, we conducted a pilot survey in a Chinese university. The immediate supervisor of students in this pilot survey was defined as an individual who is in a superior position in the organizational hierarchy and has authority and responsibility to manage students' daily activities. We collected data of all 88 items from 611 students across six departments of this university. The average age of respondents was 20.3 years (SD $=1.3)$, and $33.4 \%$ were male.

Sample 2 (88 trust items). To reproduce the factor structure of the 88 items, the main survey was conducted for Japanese employees $(n=477)$. Data was collected from all over Japan through a research company. $55.8 \%$ of respondents were male. Their average age was 38.85 years $(S D=6.45)$, average job tenure was 12.92 years $(S D=7.54)$, and the average length of time having worked with the current supervisor was 3.94 years $(S D=4.11)$. In terms of education, $26.6 \%$ had high school degrees, $24.9 \%$ had junior college or vocational degrees, $40.0 \%$ had college degrees, and $8.5 \%$ had graduate degrees. In terms of post, $41.5 \%$ were general employees, $28.9 \%$ and $25.2 \%$ were low and middle level managers, respectively, and others (4.4\%).

Sample 3. To validate CTS-6 \& ETS-6 in Supervisor, Chinese employee sample $(n=301)$ was gathered from eight cities of China: Shenzhen, Guiyang, Huainan, Qingdao, Tianjin, Shenyang, Anshan, and Jilin. $48.2 \%$ of respondents were male and $51.8 \%$ were female. Their average age was 31.39 years $(S D=7.48)$, average job tenure was 6.25 years $(S D=7.31)$, and the average length of time having worked with the current supervisor was 4.43 years $(S D=5.02)$. In terms of education, $6.3 \%$ had high school or vocational degrees, $23.9 \%$ had junior college degrees, $39.9 \%$ had college degrees, and $29.9 \%$ had graduate degrees. In terms of post, $49.8 \%$ were general employees, $18.9 \%$ were low level managers, $17.3 \%$ were middle level managers, $6.3 \%$ were top level managers, and others (7.6\%).

Sample 4. To validate CTS-6 \& ETS-6 in Supervisor, Japanese employee sample $(n=475)$ was collected from all over Japan through the same research company. 54.7\% of respondents were male. Their average age was 38.64 years $(S D=6.55)$, average job tenure was 12.90 years $(S D=7.72)$, and the average length of time having worked with the current supervisor was 4.31 years $(S D=4.76)$. In terms of education, $25.5 \%$ had high school degrees, $22.7 \%$ had junior college or vocational degrees, $42.9 \%$ had college degrees, and $8.9 \%$ had graduate degrees. In terms of post, $44.8 \%$ were general employees, $27.2 \%$ and $22.1 \%$ were low and middle level managers, respectively, and others (5.9\%).

\section{Measures in Sample 3 and 4}

All variables were measured on 7-point scale. The language expression between Chinese and Japanese version questionnaire was checked by a linguist.

Cognitive \& Emotional Trust in Supervisor. The two kinds of trust in supervisor were measured by CTS-6 \& ETS-6 in Supervisor, respectively. (1) CTS-6: Q02. My supervisor is known to be successful in both professional and social life; Q43. My supervisor will form well thought-out plans about his/her job; Q09. I believe that my supervisor would act in my best interest; Q10. If I required help, my supervisor would do his/her best to help me; Q14. My supervisor is very consistent in decisions and behaviors; Q30. My supervisor tries hard to behave on the basis of sound principles. (2) ETS-6: Q68. I would usually talk with my supervisor about personal troubles; Q71. My supervisor 
and I always talk about our work experience and opinions with each other; Q87. My values are similar with my supervisor; Q90. I have a strong sense of comradeship with my supervisor; Q58. I would be willing to ask my supervisor to solve some difficult problems for me, even if s(he) has no obligation for these matters; Q61. Faced with a difficult work situation, I would be willing to work together with my supervisor shoulder to shoulder.

Other variables. (3) Opportunities obtained in the past was measured with 2 items (Chinese $\alpha$ $=.89$, Japanese $\alpha=.90$ ). An example item was "From an objective viewpoint, I have got good chances to display my ability in this organization." (4) Altruistic behavior for supervisor was measured using 2 items (Chinese $\alpha=.93$, Japanese $\alpha=.93$ ). An example item was "I sometimes sacrifice my own interests to help the supervisor." (5) Disappointment was measured with 2 items (Chinese $\alpha=.75$, Japanese $\alpha=.63$ ). An example item was "Altogether, my expectations for my supervisor have been disappointed.” (6) Positive work attitude was measured using 3 items. (Chinese $\alpha=.75$, Japanese $\alpha=.63$ ). An example item was "I always strive for excellence in work content and process.” (7) Task dependence was measured using two items of Morgeson \& Humphrey’s (2006) WDQ (Chinese $\alpha=.70$, Japanese $\alpha=.77$ ). (8) Autonomy was measured with two-item adaptation of Morgeson \& Humphrey's (2006) WDQ (Chinese $\alpha=$.77, Japanese $\alpha=$.78). (9) $P$-O fit was measured with three items developed by Judge \& Cable (1997) (Chinese $\alpha=.63$, Japanese $\alpha=.67$ ). (10) Work-related risk was measured with five-items (Chinese $\alpha=.86$, Japanese $\alpha=.85$ ). An example item was "How much possibility is there that you and/or your organization will suffer a great loss due to the errors in your supervisor's judgments?”

\section{Results}

\section{Development of CTS-6 \& ETS-6 in Supervisor}

Validity of 2-Dimensional Structure. First, using sample 1 (as a pilot study), we conducted EFA (maximum likelihood method) to check the factor loading pattern of 88 items. In the first estimation (the number of factors was not specified), the initial eigenvalues ( $\%$ of variance) were 44.420 (50.477\%), 6.423 (7.299\%), 2.211 (2.513\%), 1.741 (1.979\%), 1.273 (1.447\%)... The scree criterion suggested that two-factor solution is appropriate for sample 1. In the second estimation (the number of factors was specified to 2 and oblique rotation was employed), overall the 43-cognitive trust items loaded on factor 1 , whereas the 45-emotional trust items loaded on factor 2 . Second, to check the reproducibility of this factor loading pattern, the same procedure was performed on the 88 items of sample 2 (as a main study). In the first estimation, the initial eigenvalues (\% of variance) were 61.485 (69.869\%), 4.456 (5.063\%), 2.279 (2.590\%), 1.597 (1.815\%), 1.040 (1.182\%)... And, the second estimation showed almost the same factor loading pattern. Thus, for both sample 1 and 2, the loading pattern of 88 items was consistent with hypothesized two-dimensional structure.

To develop scales, we selected items that (1) have theoretically important content; and/or (2) have high loading on the intended factor and low cross-loading on the other for both sample 1 and 2 from the 88 items. This procedure resulted in 22-cognitive trust items and 26-emotional trust items.

Validity of Assumed Content Domains for Each Dimension. To check the subfactor loading pattern of 22-cognitive trust items against the assumed content domains, we ran EFAs (the number of subfactors was specified to 3 and oblique rotation was employed) separately using sample 1 and 2 . For both sample 1 and 2, the subfactor loading pattern was overall not inconsistent with the three domains, except some problematic items. The same procedure was used to check 26-emotional trust items. The subfactor loading pattern was overall not inconsistent with the three domains for both sample 1 and 2, except some problematic items.

By selecting items (1) with lower cross-loading on other subfactors and/or (2) with more important content for the corresponding domain from these items, two short-version scales-6-item cognitive trust scale (CTS-6) and 6-item emotional trust scale (ETS-6) were composed. As shown in Table 1, CTS-6 consists of Q2, Q43 (competence); Q09, Q10 (benevolence); and Q14, Q30 (integrity). ETS-6 
is composed of Q68, Q71 (emotional linkage); Q87, Q90 (identification); and Q58, Q61 (depending willingness).

Table 1. Factorial Validity Evidence (Separate Estimation)

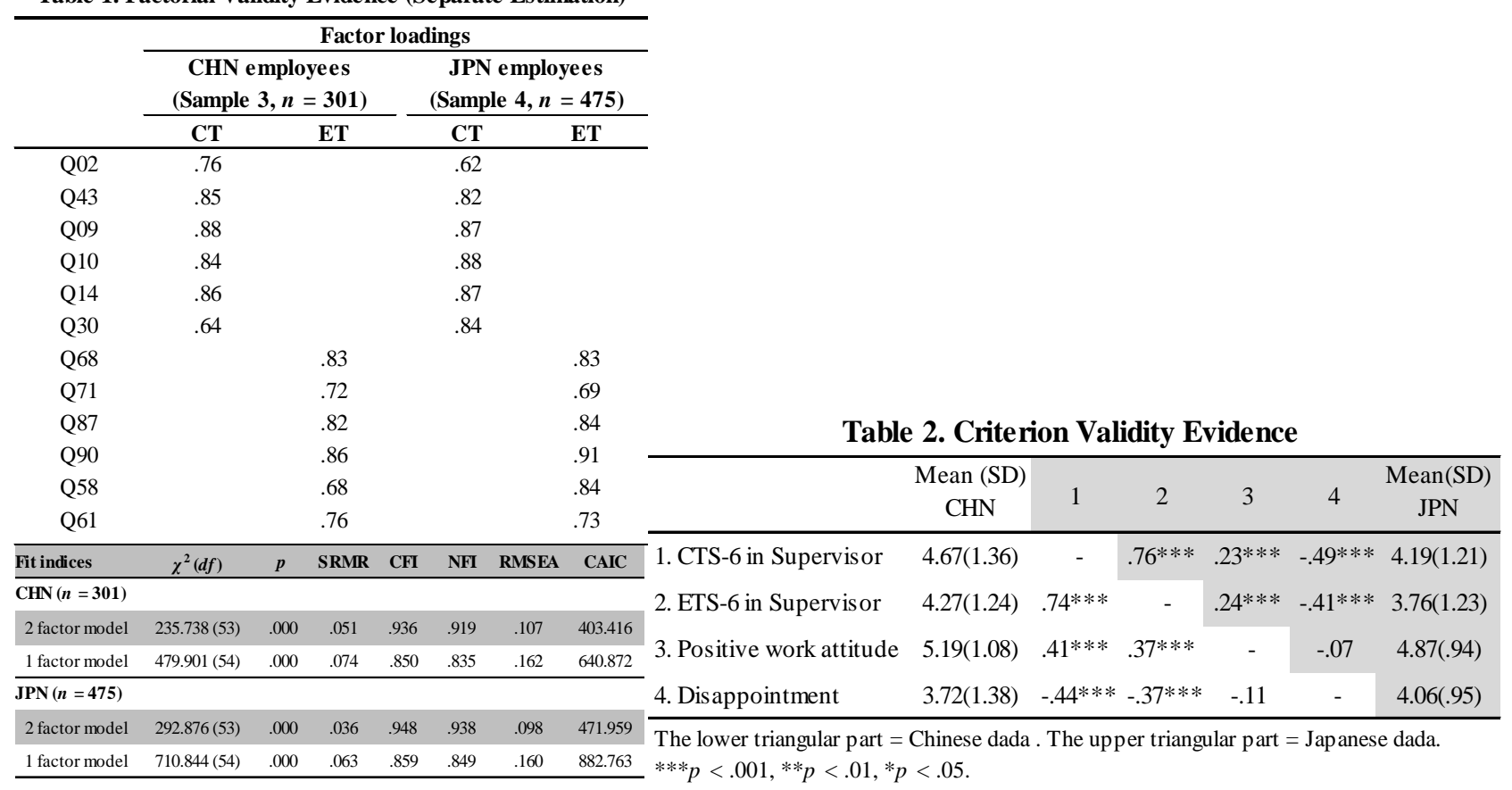

\section{Validation of CTS-6 \& ETS-6 in Supervisor}

The psychometric properties of these two scales were assessed using sample 3 and 4 . The coefficient alpha of CTS- 6 was $\alpha=.93$ for sample 3, and $\alpha=.92$ for sample 4, coefficient alpha of ETS-6 was $\alpha$ $=.90$ for sample 3 , and $\alpha=.92$ for sample 4 .

Results of Factorial Validity Analysis. (1) Factorial validity: We used CFAs to evaluate factorial validity hypothesis 1 with sample 3 and 4 separately ( $d f=53$; Table 1$)$. For both sample 3 and 4 , the hypothesized 2-factor model showed acceptable fit and was superior to the alternative 1-factor model (i.e., overall trust) in CAIC. Hence, hypothesis 1 was supported by both sample 3 and 4, indicating that factorial validity of CTS-6 \& ETS-6 in Supervisor was established. (2) Cross-cultural invariance: We further joint-estimated the hypothesized 2-factor model $(d f=106)$ to examine its cross-cultural invariance. The results $\left(\chi_{(d f)}^{2}=528.652(106), p=.000\right.$, SRMR $=.051, \mathrm{CFI}=.944$, $\mathrm{NFI}=.931$, RMSEA $=.072$ ) showed that the cross-cultural invariance of CTS-6 \& ETS-6 in Supervisor held across Chinese and Japanese sample.

Results of Convergent and Discriminant Validity Analysis. To test convergent and discriminant validity hypotheses, we run multiple regression analyses with sample 3 and 4 separately, controlling gender, age, education, post, tenure, tenure with supervisor, task dependence, autonomy, P-O fit, and work-related risk. There results indicated that convergent and discriminant validity of CTS-6 \& ETS-6 in Supervisor were consistently supported by Chinese and Japanese sample.

- For sample 3: (1) Both the effect of opportunities obtained in the past $(\beta=.15, t=3.33)$ on cognitive trust in supervisor and the effect of altruistic behavior for supervisor $(\beta=.16, t=4.25)$ on emotional trust in supervisor were significant, so that convergent validity hypotheses $\mathrm{H} 2-\mathrm{a}$ and H3-a were supported; (2) Both the effect of altruistic behavior for supervisor $(\beta=-.05, t=$ -1.24) on cognitive trust in supervisor and the effect of opportunities obtained in the past $(\beta=.07$, $t=1.56)$ on emotional trust in supervisor were not significant, so that discriminant validity hypotheses H2-b and H3-b were supported.

- For sample 4: (1) Both the effect of opportunities obtained in the past $(\beta=.10, t=2.66)$ on cognitive trust in supervisor and the effect of altruistic behavior for supervisor $(\beta=.15, t=4.89)$ on emotional trust in supervisor were significant, so that convergent validity hypotheses $\mathrm{H} 2-\mathrm{a}$ and H3-a were supported; (2) Both the effect of altruistic behavior for supervisor $(\beta=.00, t=$ -.04) on cognitive trust in supervisor and the effect of opportunities obtained in the past $(\beta=.05$, 
$t=1.55)$ on emotional trust in supervisor were not significant, so that discriminant validity hypotheses H2-b and H3-b were supported.

Results of Criterion Validity Analysis. To evaluate criterion validity the two scales, we checked their correlations with positive work attitude and disappointment using sample 3 and 4 separately. It is reasonable to expect that cognitive \& emotional trust in supervisor are positively related to employees' positive work attitude and negatively related to their disappointment. The expected pattern of positive and negative correlations was found consistently across sample 3 and 4 (Table 2), so that criterion validity of CTS-6 \& ETS-6 in Supervisor was confirmed.

\section{Discussion}

The first purpose of this paper is to develop a general conceptualization of cognitive and emotional trust, which is systematic and detailed enough to predict how they causally relate to other constructs differently. All of five middle-range hypotheses in organizational setting derived from it were supported by Chinese and Japanese samples. This fact suggests its validity and generality. The second purpose is to develop and validate middle-range scales (CTS-6 \& ETS-6 in Supervisor), which can be distinguishable enough to reliably capture their unique relations in organizational setting across different cultures. First, the reliability coefficients $\alpha$ suggests that for both Chinese and Japanese sample each scale's items have good internal consistency. Second, the testing results of factorial validity hypothesis 1 and convergent \& discriminant validity hypotheses 2 3 established construct validity of the two scales across Chinese and Japanese sample. Third, the results of criterion validity analysis established criterion validity of the two scales across Chinese and Japanese sample. These results suggest that CTS-6 \& ETS-6 in Supervisor can reliably capture the two constructs' unique relations across different cultures.

\section{References}

[1] D. McAllister, Affect- and cognition-based trust as foundations for interpersonal cooperation in organizations, Academy of Management Journal. 38, 1995, pp.24-59.

[2] Dirks, K. T., Ferrin, D. L., 2002. Trust in leadership: Meta -analytic findings and implications for organizational research, Journal of Applied Psychology, 87, 611-628.

[3] D. Rousseau, S. Sitkin, R. Burt, and C. Camerer, Not so different after all: A cross-discipline view of trust, Academy of Management Review. 23(1998)387-392.

[4] P. M. Blau, Exchange and Power in Social Life, New York: John Wiley, 1964.

[5] R. Cropanzano, M. S. Mitchell, Social exchange theory: An interdisciplinary review, Journal of Management. 31 (2005) 874-900.

[6] J. A. Colquitt, M. D. Baer, D. M. Long, \& M. D. K. Halvorsen-Ganepola, Scale indicators of social exchange relationships: A comparison of relative content validity, Journal of Applied Psychology. 99 (2014) 599-618.

[7] R. C. Mayer, J. H. Davis, F. D. Schoorman, An integrative model of organizational trust, Academy of Management Review. 20 (1995)709-734. 